Research Article

\title{
Application of Motion Sensor Fusion in Special Competitive Sports
}

\author{
Duan Xingjun \\ Department of Physical Education, Gansu Agricultural University, Lanzhou, Gansu, China \\ Correspondence should be addressed to Duan Xingjun; duanxj@gsau.edu.cn
}

Received 14 September 2021; Accepted 25 September 2021; Published 23 October 2021

Academic Editor: Guolong Shi

Copyright (c) 2021 Duan Xingjun. This is an open access article distributed under the Creative Commons Attribution License, which permits unrestricted use, distribution, and reproduction in any medium, provided the original work is properly cited.

\begin{abstract}
With the advancement of science and technology and the rapid development of the field of intelligence, new sensor technology has also made great progress. The availability and use speed of motion sensor technology are increasing rapidly, while its cost is reduced, the overall performance is also more complete, and the application fields are becoming more and more extensive. Based on the introduction and summary of the human body structure and the characteristics of sports, in order to facilitate the study of human movement, this article establishes a rod-shaped sports model and a human body coordinate system that is convenient for analysis and calculation. Through the analysis of commonly used sports parameter measurement methods, a method of using acceleration parameters for sports measurement and analysis is proposed. The motion sensor is used to measure the movement of the limbs, and the mathematical relationship between acceleration parameters and speed, displacement, and other parameters is used to solve other motion parameters, so as to analyze the posture and movement trajectory of sports. We developed a motion acquisition system which is divided into two parts: a signal acquisition unit and a data processing unit. With ARM7 and three-axis accelerometer ADXL330 as the core, it can realize information acquisition and data analysis of up to 5 sensor network nodes. The topology management platform mainly formulates the sleep strategy of suitable nodes according to the changes of the sensor network topology, maintains the connectivity of the network, and ensures the effective transmission of data. On the basis of the performance test of the system, a sports measurement experiment was carried out, and it was applied to the control of competitive sports for reliability testing. The experimental results verified the use of the sports information acquisition system to collect and analyze sports information.
\end{abstract}

\section{Introduction}

Human sports are closely related to human health and have been receiving extensive attention. With the development of sports biomechanics and anthropometry, people have increasingly studied sports [1]. Research is generally based on the theory of sports biomechanics. The measurement and analysis methods of sports parameters are studied. The main purpose is to explore the relationship between sports biomechanical parameters and reveal the laws of human motion through the measurement and analysis of sports biomechanical parameters to provide theoretical guidance in human body gesture recognition, sports training, etc. [2]. With the development and progress of social civilization, human beings pay more attention to caring for themselves. In this era, studying sports and how to coordinate the rela- tionship between people and the external environment, improve production efficiency, and improve people's working environment is obviously of far-reaching practical significance [3].

To conduct sports research, the most important step is naturally the detection of sports information, and sports detection technology is a very widely used technology produced by the integration of multiple disciplines. Its basic principles involve medicine, mathematics, and physics; computer science and other disciplines are closely related to virtual reality technology, modeling technology, computer animation technology, pattern recognition technology, human-computer interaction technology, data visualization technology, and sensor technology [4-6]. The aspects that need to be considered when acquiring athlete information outside the laboratory environment are severely affected 
and dependent on currently available technologies. Through technological innovation and development, new sensor equipment and systems will provide the possibility for them. At the same time, in terms of size and power, the reduction in demand and cost will also become a general trend [7]. Sports biomechanics is based on the knowledge of kinematics, dynamics, human anatomy, etc. and uses anthropometric technology to collect information. The system mechanics of sports organs and the human body perform partial or overall movement in space. Under the action, the human body's position, posture, movement speed, and other changes in time and space are studied [8]. In the field of sports, obtaining the biomechanical information of athletes' technical movements enables us to shape the standard model of sports technology, explore ways to optimize sports technology, or diagnose the athletes' current technical movements to improve training. The process enables outstanding athletes to make breakthrough progress in technical movements and create excellent sports performance [9].

This paper analyzes sports, especially the relationship and characteristics of sports and sports acceleration parameters. On the basis of research and analysis of a large number of documents, the human body is regarded as a combination of various links connected by joints, and a stick figure model of the human body is established. Through the establishment of sports coordinate system, we study the movement of each feature point of the limbs. In this paper, the hardware design of the entire sports information collection system based on motion sensors is completed, and the overall design plan of the system is given first, and on this basis, the system adopts a modular design and the attitude measurement unit, UWB positioning unit, and data storage and communication unit. This new type of sensor is a combination of a barometer and a three-axis accelerometer, which can more accurately measure the energy consumption of vertical motion. The attitude measurement unit is designed according to different needs in actual use of reliability, separately designed data offline storage unit, and Bluetooth remote communication unit. The system adopts a modular design, which makes the system more convenient to expand and upgrade and has better applicability and reliability.

\section{Related Works}

With the advancement of science and technology and the rapid development of the field of intelligence, new sensor technology has been greatly improved, and the application field has become more and more extensive, especially in the field of sports. It can safely and effectively provide data, promote the development of sports, and meet the needs of coaches and athletes for the needs of the instrument [10]. In terms of sports physiology and mechanics, computer simulation of sports is a higher level of research content and an effective means to study the laws of sports; it has high theoretical and practical application value, such as simulation of collisions and human analysis for motor characteristics, neurosurgery diagnosis in medical treatment, and gait research. Of course, there are many methods and methods to study sports. Among them, the experimental method is a very promising research method. Making full use of the test data obtained from the experiment can simplify the complexity of human body modeling, shorten the research period, and simulate sports examples [11-13].

Xia et al. [14] founded the kinetic measurement technology based on sports efficiency, and later generations have further developed muscle strength optimization technology and energy optimization technology. After the application of high-speed photography technology, sensor measurement technology, and synchronous measurement technology to the measurement of sports biomechanics, the research of sports biomechanics measurement has entered the level of three-dimensional kinematics and spatial mechanics, providing a comprehensive study of human exercise performance and good guarantee. Heath et al. [15] obtained the support of experimental technology while solving the problem of human kinematics. Especially, the infiltration of contemporary computer graphics, video animation technology, and motion tracking and capturing technology has greatly enriched the measurement technology of sports biomechanics. With the development of MEMS (microelectromechanical sensor) technology and semiconductor technology, the miniaturization and intelligentization of acceleration sensors have made them more widely used in sports detection. Pueo [16] found that in the analysis of the current research status in the article "The Application of Accelerometer in Sports Detection," combined with the relevant research in recent years, from the aspects of sports training, medical clinical auxiliary diagnosis, sports simulation research, etc., motion sensors are used in sports detection.

Foreign researches on the application of motion sensors to sports training are relatively leading. Earlier, Mendes et al. [17] used a piezoresistive three-axis motion sensor to measure the relationship between sports acceleration and energy consumption to realize the measurement of the acceleration of the human body in daily activities such as sitting and walking, and then obtain the relationship between the acceleration value and the energy consumption of sports from the experiment. From the analysis of the measurement results, the acceleration value can reflect a certain exercise intensity of the measured human body. Feng and Tan [18] analyzed the performance of a "sensitive armband" that can perceive and obtain information about daily life activities such as standing, sitting, walking, running, jumping, and riding and can be worn on the arm. Some scholars have also mentioned in their research that the increase in distance will result in greater space limitations for shooting, which requires players to adjust the accuracy and impulse of releasing the ball. The decrease in the height and angle of the toss and the increase in the speed of the toss are considered to be the main factors that reduce the accuracy of the toss with the increase in distance [19]. Some scholars have compiled the literature and concluded that the factors affecting the stability of free throws are subjective and objective. The subjective aspects mainly include technical aspects, physical conditions, and psychological quality. The objective aspects mainly include environmental influences and external pressures. In view of these influencing factors, it also summarizes 
cutting-edge training concepts and methods at home and abroad. The article emphasizes that technical training should pay more attention to details and psychological training. Training methods to improve the stability of free throws include basic technical training refinement, appearance training method, implied training method, programmed behavior training method, and conditional restriction method [20, 21].

\section{Construction of Application Model of Motion Sensor Fusion in Special Competitive Sports}

3.1. Motion Sensor Parallel Architecture. The general motion sensor utilizes the characteristic of crystal deformation caused by acceleration. Since this deformation generates a voltage, as long as the relationship between the generated voltage and the applied acceleration is calculated, the acceleration can be converted into a voltage output. Figure 1 shows the parallel architecture of the motion sensor.

The analog output voltage is generally proportional to the acceleration. For example, for a motion sensor with a sensitivity of $500 \mathrm{mv} / \mathrm{g}$, when $2.5 \mathrm{~V}$ corresponds to an acceleration of $2.6 \mathrm{~V}$, it corresponds to an acceleration of 0.59 . The digital output generally uses a pulse width modulation (PWM) signal.

$$
\begin{aligned}
& X[t]=\{t \in R \mid x(1), x(2), \cdots, x(t)\}, \\
& y(n)=A \times X(n)-t \times x(n-1) .
\end{aligned}
$$

The minimum acceleration measurement value is also called the minimum resolution. Considering the noise of the postamplification circuit, it should be kept as far away as possible from the minimum available value.

$$
\overline{x^{2}}(t, n)-|x(t, n)|^{2}-2 \overline{|x(t, n)|} \times \cos \alpha=0
$$

The maximum measurement limit should consider the nonlinear effect of the motion sensor itself and the maximum output voltage of the subsequent instrument. For the estimation method, maximum measured acceleration $x$ sensor charge (voltage) sensitivity, whether its value exceeds the maximum input charge (voltage) value of the supporting instrument.

$$
T(x, y, \theta)=\left(\begin{array}{ccc}
\frac{\partial^{2} \alpha}{\partial x^{2}} & \sin \theta & 0 \\
\sin \theta & \frac{\partial^{2} \alpha}{\partial y^{2}} & \cos \theta \\
0 & \cos \theta & \frac{\partial^{2} \alpha}{\partial x \partial y}
\end{array}\right)
$$

After the analog signal output by the motion sensor is filtered and processed, the analog-to-digital conversion is required to process the data. The A/D conversion link adopts the 8-bit A/D converter inside the control chip ATmega48 of the slave. The slave of the signal acquisition unit is composed of the motion sensor ADXL330, signal conditioning circuit, single-chip ATmega48, and related peripheral circuits. The master of the signal acquisition unit forms a network with all slaves through the $12 \mathrm{C}$ bus and controls the slave to complete the signal conversion and transmission.

$$
\nabla^{2} f(x, y)=\frac{\partial^{2} f(x, y)}{\partial x \partial y}+\frac{\partial^{2} f(x, y)}{\partial x \partial y}
$$

The internal structure refers to the way that the sensitive material crystal piece feels vibration and the installation form. There are two types of compression and shearing, and the common ones are central compression, plane shear, triangular shear, and ring shear.

$$
\begin{aligned}
G(x, y)= & \left\{(\sqrt{f(x, y)}-\sqrt{f(x+1, y+1)})^{2}\right. \\
& \left.+(\sqrt{f(x+1, y)}-\sqrt{f(x, y+1)})^{2}\right\}^{1 / 2}, \\
H(x, y)- & \nabla^{2}(G(x, y) \times f(x, y))=0 .
\end{aligned}
$$

The center compression type has a higher frequency response than the shear type, and the shear type has better environmental adaptability than the center compression type. If it is equipped with an integral charge amplifier to measure speed and displacement, it is best to choose a shear type product, so that the signal obtained has small fluctuations and good stability.

3.2. Sports System Integration. The movement system is composed of bones and muscles fixed on the bones. Under the control of the nervous system, the muscles contract and relax, which affect the bones and enable the human body to perform various movements. The essence is that the limbs move around the joints. Therefore, the human body can be regarded as a combination of multiple rigid bodies. The joints are connected to form a connected rigid system. Because all the movements of the human body are realized through joint movement, the most important thing is to analyze the degree of freedom of each joint, the corresponding motion range, and the constraints and linkage between multiple joints. The energy management platform is responsible for managing the energy usage of the nodes, so that the nodes can cooperate to complete the monitoring tasks under low energy consumption and coordinate the operation of the nodes to avoid repeated consumption. The basis of sports is the rotation of the links around the joint axis. Generally speaking, joint and link motions refer to the rotation of the links around the joint axis. Table 1 shows the distribution of joint rotation attributes. Joint rotation is divided into five types according to the type of rotation axis and the direction of rotation: namely, flexion and extension, horizontal flexion and extension, adduction and abduction, rotation (pronation, supination), and circular rotation (circumflexion). 


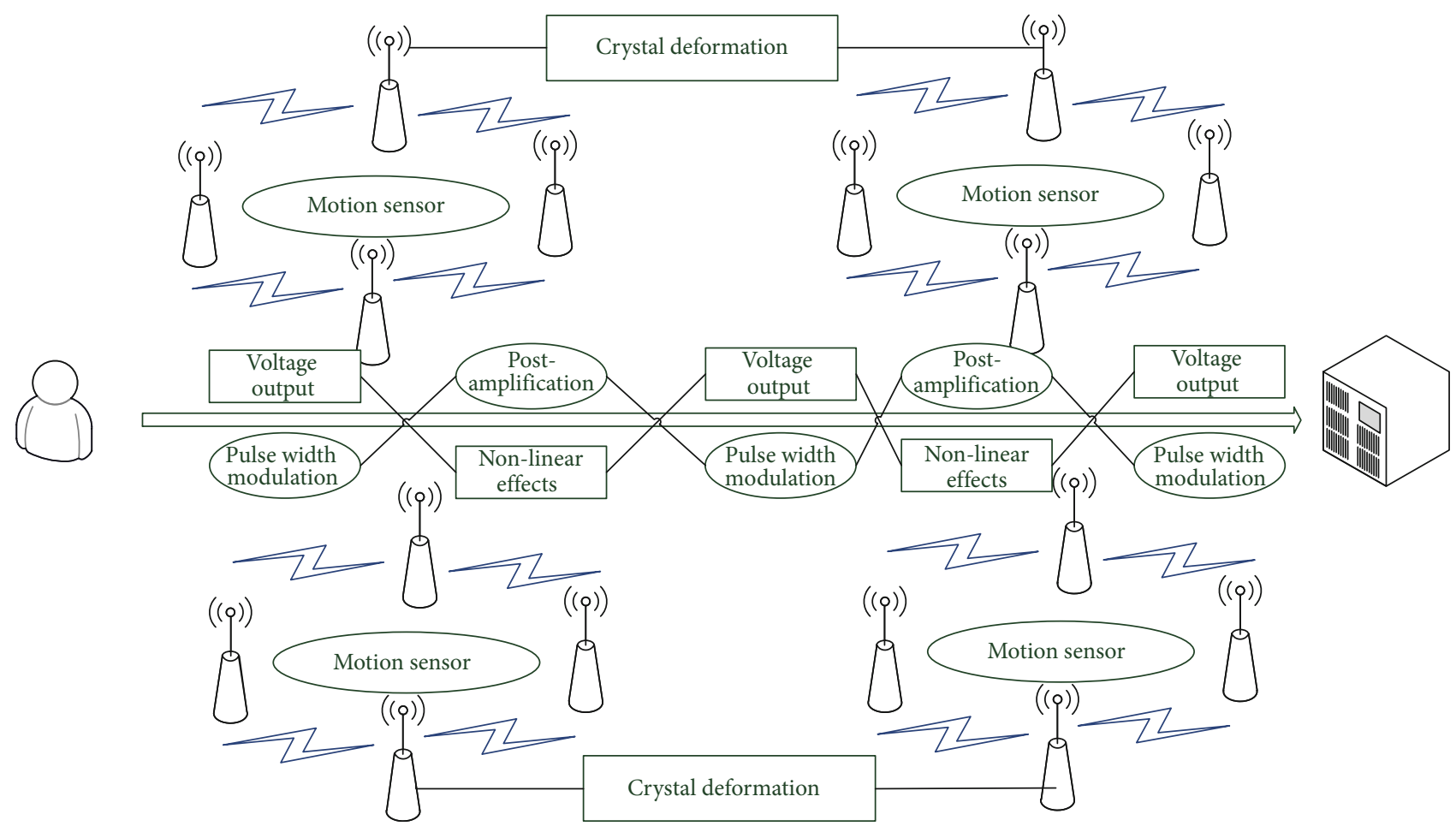

FIGURE 1: Motion sensor parallel architecture.

TABle 1: Distribution of joint rotation attributes.

\begin{tabular}{lccc}
\hline Attribute index & Heart rate & Height $(\mathrm{cm})$ & Running speed $(\mathrm{km} / \mathrm{h})$ \\
\hline 1 & 72 & 191 & 5.7 \\
2 & 69 & 188 & 8.4 \\
3 & 81 & 192 & 8.7 \\
4 & 77 & 194 & 9.1 \\
\hline
\end{tabular}

From the perspective of cognition, sports can be divided into three different levels: action, action (activity), and basic action (movement). Among them, the basic movement is the most basic unit of movement, which can be represented by the spatiotemporal trajectory of the human joints over a period of time. In the real world, even if the same person repeats a certain basic action, the trajectory of the action cannot be completely the same, so it is difficult to have a strict mathematical definition of the basic action. Only when a specific scene is given and the movements in the scene can be compared and classified can it be said that the movement classified into one category is a basic action. Action is a movement defined on top of basic actions. It is a combination of some basic actions. Unlike basic actions, the same type of action may be composed of different basic actions; that is, their trajectories may be very different. And behavior mainly refers to actions that contain the exact semantic meaning related to a specific environment.

3.3. Parameter Optimization of Competitive Sports Model. The motion accelerometer in the attitude measurement unit can rely on a high-precision three-axis turntable and use the gravity acceleration vector roll method for calibration.
Figure 2 shows the accelerometer sensitivity output result. We run the high-precision three-axis turntable control program, so that each sensitive axis direction of the three-axis motion accelerometer installed on the three-axis turntable can be sequentially sensitive to the input of gravitational acceleration in different directions, that is, the carrier coordinate system $x$-axis upward, the carrier coordinate system $y$ -axial up, carrier coordinate system $z$-axis up, carrier coordinate system $x$-axis down, carrier coordinate system $y$-axis down, and carrier coordinate system $z$-axis down, and record the raw data output by the three-axis motion accelerometer. The entire calibration process of the axis motion accelerometer needs to record the accelerometer output data in 6 cases.

A total of 16 sets of motion sensor output data need to be stored for one experiment, and a total of 3 experiments are carried out. In one experiment, the temperature control box starts from the highest temperature and ends at the highest temperature. The transport layer is responsible for the transmission control of the data stream to ensure the quality of service. It divides the data stream received from the application layer into fixed units and then transmits it to the network layer to perform flow control and congestion control and ensure the reliable and orderly arrival of data. The experiment process is similar to the other two experiments. We open the high-precision three-axis turntable control program, use the rate mode to provide the specified angular rate input for the axis to be measured, and use the positioning mode to keep the other axes stationary to prevent the quadrature axis angular rate input from affecting the measurement. The coordinate system consists of the body coordinate system, the shoulder coordinate system, 


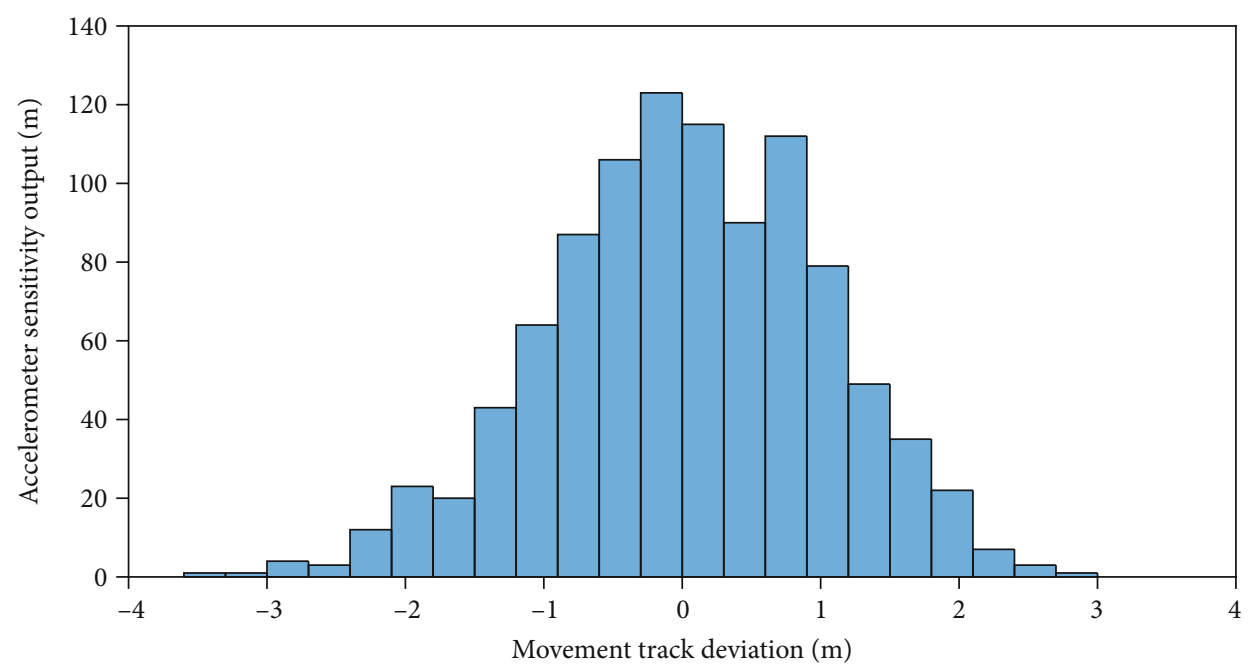

FIGURE 2: Sensitivity output result of accelerometer.

the elbow coordinate system, and the wrist coordinate system. The position of the human shoulder in the body coordinate system (D system) is recorded as $z$. It represents the projection of the three-dimensional vector of the origin of the shoulder coordinate system relative to the origin of the body coordinate system in the body coordinate system. The position of the elbow in the shoulder coordinate system (S system) is marked as bracket $e$, which represents the projection of the three-dimensional vector of the origin of the elbow coordinate system relative to the origin of the shoulder coordinate system in the shoulder coordinate system. The position of the wrist in the elbow coordinate system (E system) is recorded as $p$, which means the projection of the three-dimensional vector of the origin of the wrist coordinate system relative to the origin of the elbow coordinate system in the elbow coordinate system.

3.4. Data Measurement Network Design. Sports information collection and analysis refer to the use of certain methods to track and capture the human body's movement, obtain some of the parameters of the human body's movement, and analyze and process these parameters, so as to reconstruct the structure and posture of the human body or perform other applications. The movement information of the human body during the movement process is mainly reflected in the body posture, joint angle, body and limb displacement, speed and acceleration, force magnitude and direction, and dynamic force change rate. By measuring this information and analyzing it with biokinematics technology, the state of the human body during exercise can be evaluated. The process of sports analysis is mainly composed of sports tracking measurement, signal processing, and data analysis. The data processing unit mainly completes the analysis and processing of the multichannel acceleration data collected by the signal acquisition unit, which can realize the system function setting, data storage, and communication with the signal acquisition unit and the host computer. Figure 3 shows the data measurement network design diagram. The hardware composition of the data processing unit mainly includes
CPU unit, memory expansion circuit, input and output circuit, communication circuit, clock circuit, and power supply circuit.

In order to analyze the collected image information, it needs to be digitally processed. For the motion information obtained by high-speed photography, the film analyzer is generally used to complete the digitization process of the film and analyze the image captured by the camera by the projector. After magnification, it is projected onto the digitizing board, and then the required coordinate value $(x, y)$ is taken out by the cursor keyboard and input into the computer for storage or data processing. The digital analog-todigital conversion of the film generally adopts two principles; one is to use the principle of ultrasonic testing, and the other is to use the principle of inductosyn. The mobility management platform is mainly responsible for monitoring and controlling the movement of nodes, recording the location of nodes, and enabling nodes to dynamically track target information. We read the data of the built-in thermometer of the motion sensor through the serial port and record the output data of the motion sensor after the data is basically stable. When the temperature reaches the maximum temperature, keep the maximum temperature. After a certain interval, we turn off and restart the motion sensor. After the output data of its built-in temperature sensor is stable, record the motion sensor output and start the cooling process experiment until it drops to the minimum temperature.

\section{Analysis of the Application Model of Motion Sensor Fusion in Special Competitive Sports}

4.1. Sensor Data Collection. The input and output circuit of the system is mainly used for the user to input instructions or perform function settings and output and display various related information. The input part adopts the key-press mode and is directly connected to the I/O port of the ARM. There are four function keys, which are defined as set, increment, decrement, and confirm; the output part 


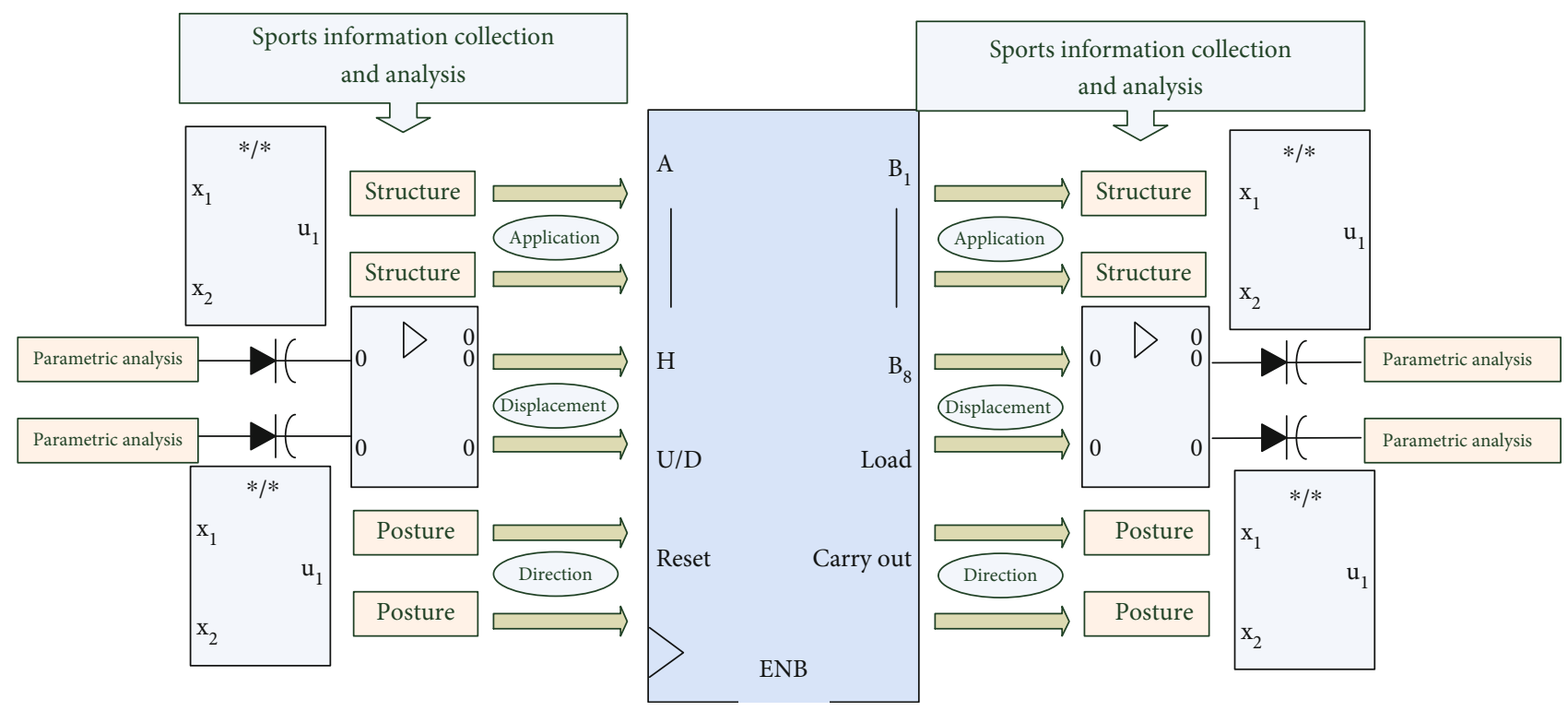

Figure 3: Data measurement network design diagram.

includes LED indicator light and LCD dot matrix liquid crystal display of model RTl2864M. Since the data processing unit needs to process and analyze 15 channels of acceleration data in this system, it has higher requirements on the CPU's computing speed and processing capacity. In this design, the ARM7 chip STR710F produced by the semiconductor company is selected. For Z2T6, the highest frequency of the processor can reach $50 \mathrm{MHz}$ and has embedded $256 \mathrm{~KB}$ F1ash, $64 \mathrm{~KB}$ SRAM, and I/O ports that include three 16-bit ports $\mathrm{PO}, \mathrm{P} 1$, and $\mathrm{P} 2$; other peripheral interface pins are connected with $\mathrm{I} / \mathrm{O}$. This window segmentation technology is relatively simple, does not require additional processing of motion signals, and is more commonly used in behavior recognition that requires high real-time performance. The pin functions of the port are multiplexed. Figure 4 shows the linear fit of the port deviation output results.

The experiment uses the temperature characteristic curve of the zero bias obtained from the output data of the MEMS gyroscope in the temperature test drawn by MATLAB. From the Figure 4, it can be seen that the zero bias of the gyroscope changes when temperature changes during the entire temperature test, and the changes are obvious. From the experimental data, it can be found that the bias change trend of the gyroscope is basically the same, but the specific values are slightly different. Therefore, the bias value of the MEMS gyroscope at different temperatures can be approximated by the difference algorithm and brought into the MEMS gyroscope. In the output model of the instrument, the corresponding angular rate information is obtained to improve its measurement accuracy.

4.2. Competitive Sports Information Extraction. In order to ensure the reliability of data collection, in the experiment, the six behaviors corresponding to each part were collected three times. The wireless inertial sensor node is mainly composed of accelerometer and gyroscope. The accelerometer model is ADXL326, and its measurement range is \pm 169 . The gyroscope models are LPR550 and LY550, and its measurement range is $\pm 500 \mathrm{dps}$, which fully meets high-speed sports such as badminton. The wireless inertial sensor node transmits data to the personal computer terminal through the wireless receiving node. Therefore, each volunteer in the experiment needs to collect 54 data times, and the time for each data collection is 2 minutes, to ensure that the collected data is not affected by the wearing of the instrument in the experiment; we check the wearing position of the instrument before each data collection; that is, check the data output of the instrument in the static state of the volunteer before each data collection. If the data changes, it will be corrected.

The frequency range of the main body part of the human body is between 0 and $20 \mathrm{~Hz}$, and its acceleration component generally does not exceed $\pm 6 g$. Therefore, in order to provide as much as possible the detailed information of sports and not occupy too much storage space, the sensitivity of the sensor is $200 \mathrm{mV} / \mathrm{g}$ (the range at this time is \pm 69 ), and a sampling frequency of $50 \mathrm{~Hz}$ is used. The system detects the body motion signal of the human body through the three-dimensional motion sensor, the signal enters the microprocessor after filtering and conditioning circuit, and the analog signal is converted into a digital signal through the own A/D converter. After further processing, it is sent to store in the static memory. The data is sent to the PC through RS232 for data processing and algorithm analysis, and then, sports energy and physical state are obtained. In order to facilitate data collection, the system adopts two power supply modes: button battery and DC power supply. When collecting data, button battery can be used for offline use.

When the human body walks, the vertical component of the frequency of the upper body part is mainly concentrated in it. The range is $8 \mathrm{~Hz}$ to $5 \mathrm{~Hz}$ (the amplitude range is from 0.39 to 0.89 ), and the horizontal component amplitudes of 


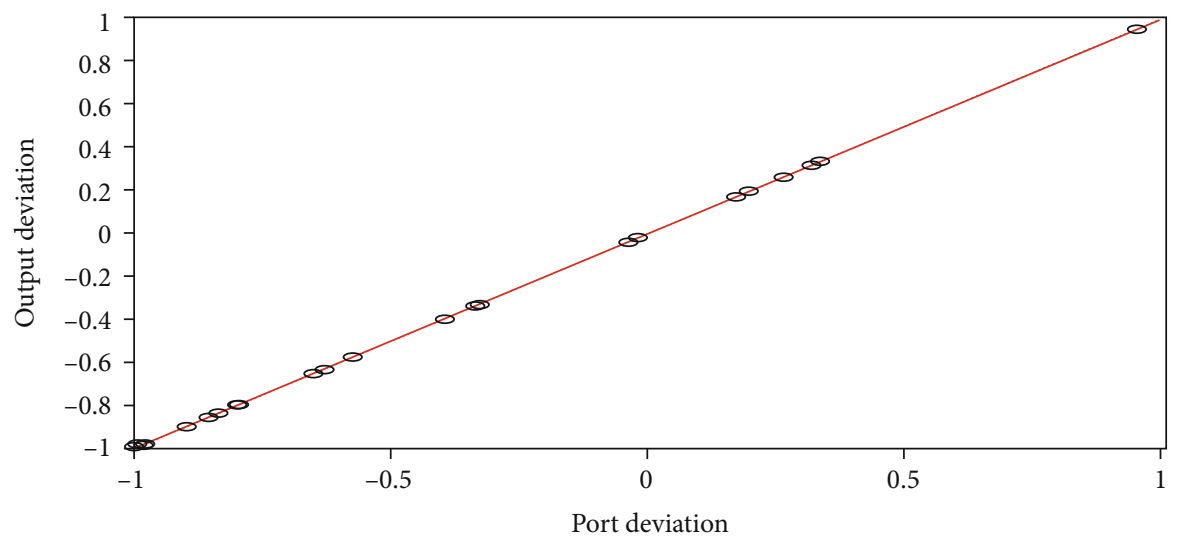

FIgURE 4: Linear fitting of port deviation output results.

the trunk and head positions are from 0.39 to 0.49 and from 0.29 to 0.99 , respectively. When the human body is running, the vertical component of the frequency at the ankle is mainly concentrated in the range of $1 \mathrm{~Hz} \sim 18 \mathrm{~Hz}$ (the amplitude range is 3.01 12.09); while the amplitude of the vertical component of the trunk part and the head position is, respectively, 0.99-5.09. Figure 5 shows the distribution trend of the vertical component of frequency. It can be found that the accelerometer measurement curve of the action has obvious characteristics. The accelerometer measurement data of the three actions of the forehand catching the backcourt, the forehand picking the backcourt, and the backhand picking the backcourt, which are not easy to distinguish with the gyroscope measurement data, are obviously different. Normalize all the obtained features, the normalized interval is $[0,1]$, and finally use PCA to reduce the dimensionality, the threshold is set to $99 \%$, and the first three principal components pca1, pca2, and pca3 of 3 levels are obtained as threedimensional distribution. Therefore, real-time or offline measurement of badminton technical movements can be carried out through the MEMS-based sports information collection system, and the data obtained can show the characteristics of the movement, which is convenient for further identification and analysis of the movement and then realizes the training and competition process of the badminton.

4.3. Realization of Motion Sensor Fusion Simulation. MMA7260 reduces the chip size by adopting $6 \mathrm{~mm} \times 6 \mathrm{~mm}$ $\times 1.45 \mathrm{~mm}$ QFN package and integrates signal conditioning, single-pole low-pass filter, and temperature compensation technology. It saves a lot of energy by providing sleep mode, so MMA7260 is a portable device choice. In addition, as a three-dimensional motion sensor, MMA7260 has the function of monitoring three-axis acceleration, so that the system can easily obtain changes in position, posture, and motion. It contains two surface micromachined capacitive sensing cells and a signal adjustment application-specific integrated circuit. The cell sensor senses the acceleration in three mutually orthogonal directions, $X, Y$, and $Z$, and the acceleration is output as a voltage signal after a voltage-capacitive converter, gain amplification, filter, and temperature compensation. Figure 6 shows the two-dimensional distribution of voltage signal output. Because the output of MMA7260 is analog voltage, it needs to carry on signal conversion through A/D that MSP430F149 comes with. Considering that the voltage at the output of the MMA7260 may be greater than the reference voltage at the input of the $\mathrm{AD}$, a voltage divider circuit is added between the two, which not only solves the problem but also maintains the shape of the original signal waveform.

The subject entered a well-known fitness center in the city to run on a treadmill to measure energy consumption. The wearing position of the instrument and the precautions of the experiment were similar to the action recognition, and the experiment was still carried out with three more common parts (buttocks, waist, and knees). In order to determine the speed as the influence of the system, we conducted three sets of energy consumption test experiments with different speeds for each part. According to the wearing position and three different speeds, the experiment is divided into nine groups. The multisensor synchronous test method can realize the synchronous test function of the miniature attitude measurement unit with high efficiency and high reliability. In its hardware design, the circuit connection board adopts the conductive spring pin contact connection method to connect with the component under test, which avoids the test. The welding and other processes cause unnecessary damage to the component under test, and the design of the circuit connection board is relatively simple and easy to replace. This method can complete the calibration, temperature compensation, and testing of 16 miniature posture measurement units at one time. After the test, the miniature attitude measurement unit has high measurement accuracy.

4.4. Case Application and Analysis. The speed signal enters the AD input terminal of the microprocessor for further processing after filtering, voltage division, and other processing. In order to store data offline, this system chooses Atmel's survival static memory for data storage. The working voltage of mode is $2.5 \mathrm{~V} \sim 3.6 \mathrm{~V}$. It can provide sleep mode to save energy consumption. In addition, it can also achieve continuous operation by alternately using two internal SRAM page 


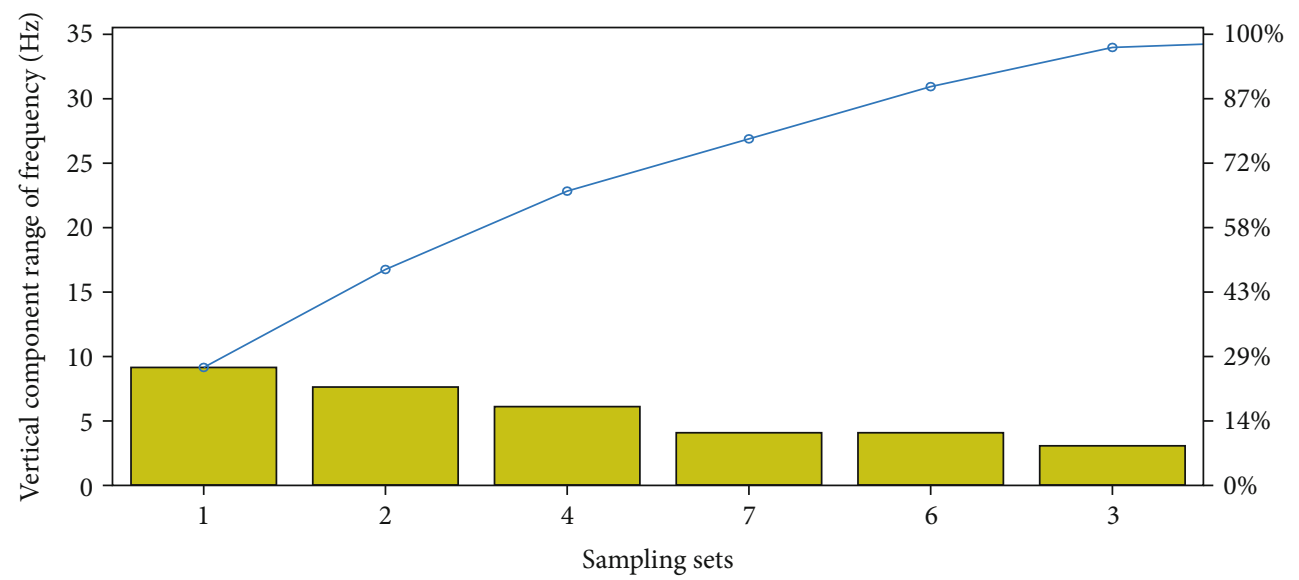

Figure 5: Vertical component distribution trend of frequency.

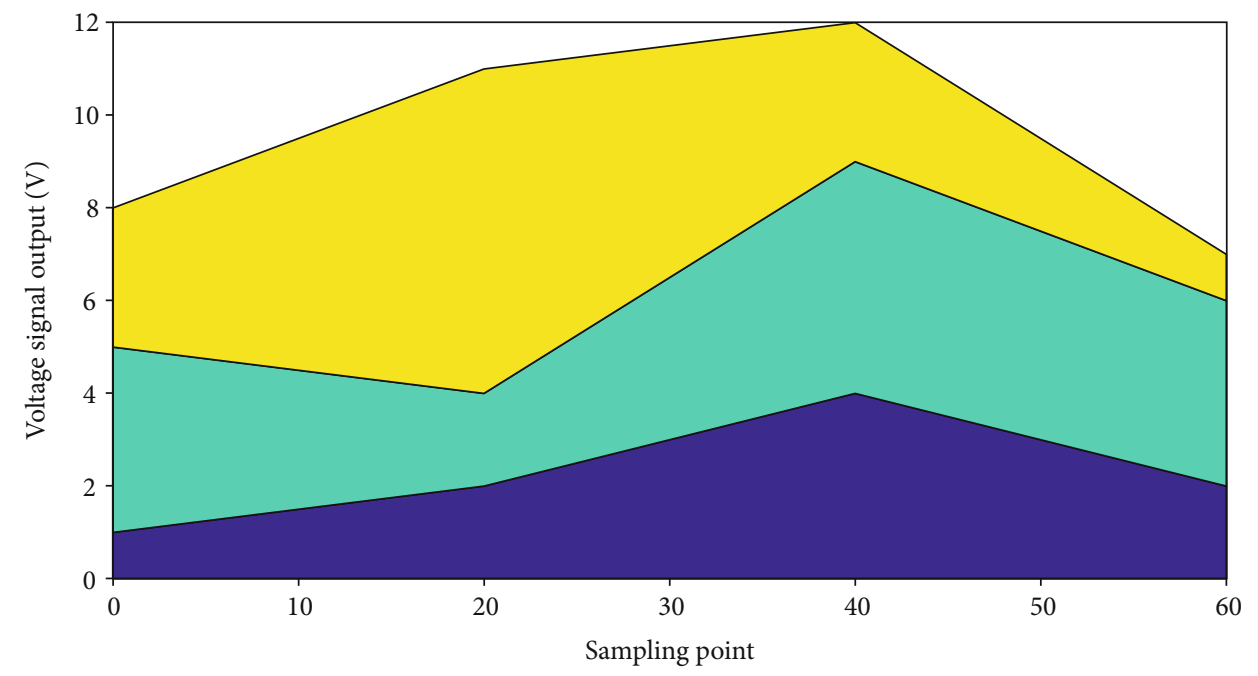

FIGURE 6: Two-dimensional distribution of voltage signal output.

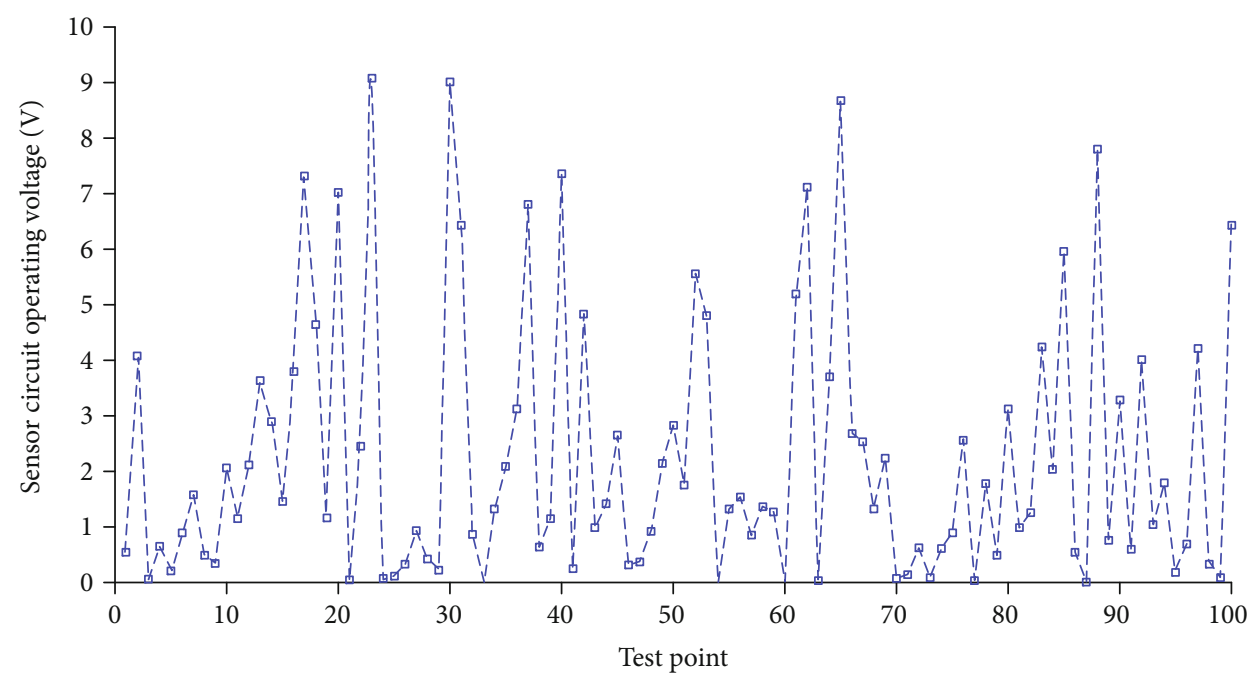

FIGURE 7: The broken line diagram of the operating voltage of the sensor circuit. 


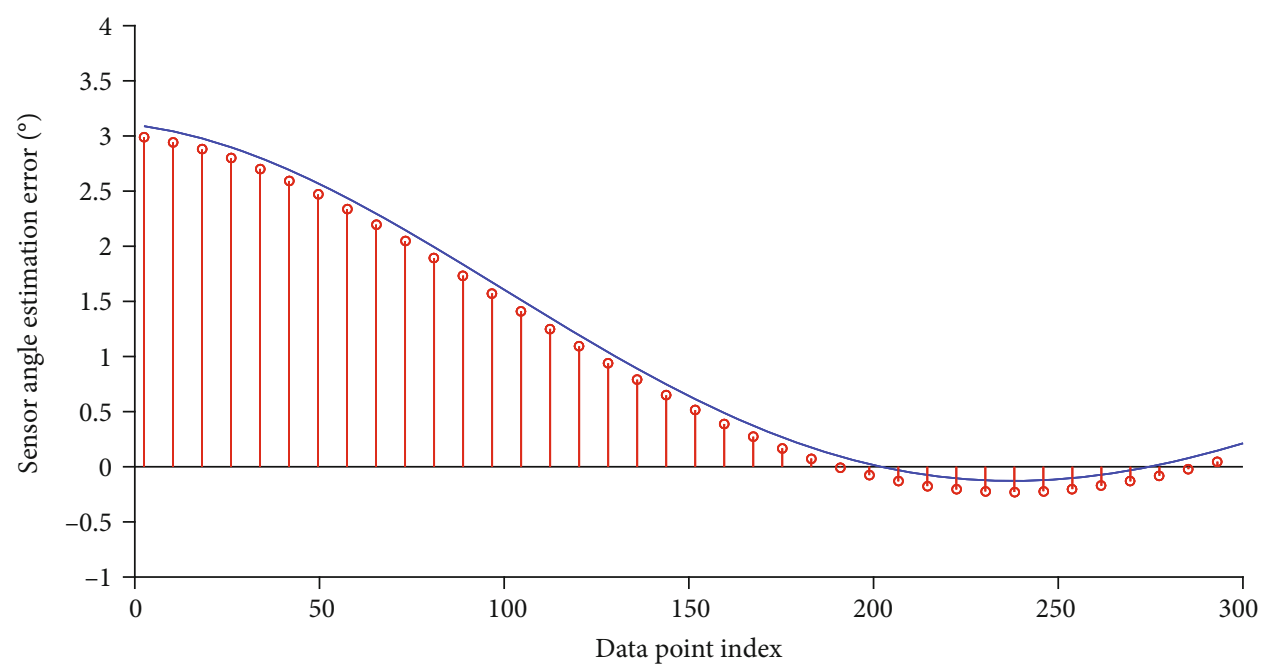

FIGURE 8: Error curve distribution of sensor angle estimation.

buffers. Figure 7 shows the line graph of the operating voltage of the sensor circuit.

The data analysis in this paper is to carry out statistical analysis in SPSS21.0 and Excel and to carry out statistical analysis and verification of the research data to ensure the authenticity and reliability of the data. The data collected by the sensors is analyzed by the professional analysis software system EMGworks. The analysis software system EMGworks can sort out the values of the three-axis acceleration when the player shoots. We calculate the maximum acceleration generated by the player ten free throws or jump shots. The results show that the attitude estimation algorithm based on the rotating coordinate system can effectively suppress the pitch and roll angle estimation errors when the pitch angle is close to \pm 90 , but the estimation error of the pitch angle and the roll angle is relatively large when the pitch angle is about 0 . The estimation error characteristics of the two attitude estimation algorithms can be complementary. In order to better achieve the accuracy of the attitude angle calculation in the full angle range, the pitch angle threshold is set and the two attitude estimation algorithms are comprehensively used. It can be seen that when only the angular velocity data is used for grade classification, the recognition rate can reach $99.3 \%$, which verifies the feasibility of the above five indicators from another aspect. This article has also done an experiment using acceleration and angular velocity data to classify grades at the same time. The recognition rate can reach $100 \%$. Once again, acceleration and angular velocity can be used to classify grades. That is, when the elevation angle is less than the threshold, the attitude estimation algorithm based on extended Kalman filter is used, and when the elevation angle is greater than the threshold, the attitude estimation algorithm based on the rotating coordinate system is used to reduce the attitude calculation error in the full angle range. Figure 8 shows the error curve distribution of sensor angle estimation.

Through the above analysis of the characteristics of the action data, it can be found that the action data has some obvious characteristics. The action category can be judged by the method of rule learning, decision tree, etc., but its rule characteristics are difficult to summarize, so the MEMSbased sports information collection system such as neural networks will be used to learn from the data. The above has completed the MEMS-based sports information collection system for the information collection test of 5 common simple actions. We continue to use badminton as the background to collect a variety of technical actions in badminton and briefly analyze the collection results. Theoretically, when the gravitational acceleration in a certain axis direction is zero, its output should be half of the supply voltage. The motion sensor itself is in a gravitational acceleration environment, so when it is stationary, if the acceleration vector in a certain direction is parallel to the gravitational acceleration vector, the acceleration value in that direction should be $\pm 1 \mathrm{~g}$. The acceleration vector is perpendicular to the gravitational acceleration vector, and the acceleration value of the sensor in this direction should be 0 . Through the analysis and comparison of the experiment and data of the two algorithms and the wearing positions of the three instruments, the best solution is finally determined to realize the correct recognition of the six actions. This system uses this characteristic of the motion sensor to calibrate the sensor system. Since the sensitivity of the sensor used in the system experiment is $200 \mathrm{mV} / \mathrm{g}$, the calibration experiment uses a sensitivity of $200 \mathrm{mV} / \mathrm{g}$, and the system uses a voltage of $3.3 \mathrm{~V}$.

\section{Conclusion}

In this paper, a three-dimensional acceleration monitoring system is built by using the three-dimensional motion sensor MMA7260 and the processor to monitor sports energy consumption and daily behavior. At the same time, the human body's energy consumption is detected. Through the experiment and data analysis and comparison of the two algorithms and the wearing position of the three instruments, the best solution is finally determined. The experimental results show that the energy consumption measurement accuracy can reach more than $94 \%$. In this paper, based on 
the main application of the sports information collection system, the motion sensors required by the system are modeled and analyzed, the wireless sensor network node and base station hardware structure are calculated, and related system software is developed, including the work of nodes and base stations. In order to ensure its measurement accuracy, a calibration method is given, and temperature compensation is carried out for the characteristic that the measurement accuracy of the motion sensor is easily affected by temperature. Furthermore, a multisensor synchronization test method is given to improve the calibration, temperature compensation, and test efficiency of the motion sensor. Finally, we use this system to collect the movement information of common head movements and badminton technical movements in life and conduct a brief analysis of the data. For the measured movements, the measurement data can express the movement characteristics and have the information collection function, which can be used. The data further implements applications such as action recognition. At the same time, the human body's energy consumption is detected, and the best solution is finally determined through the experiment and data analysis and comparison of the two algorithms and the wearing position of the three instruments. The experimental results show that the energy consumption measurement accuracy can reach more than 94\%. Experimental results show that by applying motion sensors to basketball events, coaches can better discover some potential sports athletes for training and training based on the stability data they provide, and they can also apply motion sensors to sports athletes' daily routines. During training, it helps players to better correct their shooting techniques and improve the shooting stability of sports athletes.

\section{Data Availability}

All data, models, and code generated or used during the study appear in the submitted article.

\section{Conflicts of Interest}

The author declares that there is no conflict of interest. Duan Xingjun is a master working on physical education in the Gansu Agricultural University. His research interests include college students' physical education and traditional ethnic minority sports, and he had more than 10 papers published and 2 books published.

\section{References}

[1] J. Yang, H. Xia, Y. Wang, and H. Tian, "Simulation of badminton sports injury prediction based on the internet of things and wireless sensors," Microprocessors and Microsystems, vol. 81, p. 103676, 2021.

[2] B. Barshan and A. Yurtman, "Classifying daily and sports activities invariantly to the positioning of wearable motion sensor units," IEEE Internet of Things Journal, vol. 7, no. 6, pp. 4801-4815, 2020.

[3] M. P. Wilk, M. Walsh, and B. O'Flynn, "Multimodal sensor fusion for low-power wearable human motion tracking sys- tems in sports applications," IEEE Sensors Journal, vol. 21, no. 4, pp. 5195-5212, 2021.

[4] T. Nagata, N. Nakamura, and M. Miyatake, "VO 2 estimation using 6-axis motion sensor with sports activity classification," Engineering in Medicine and Biology Society, pp. 4735-4738, 2016.

[5] C. Zhang, F. Yang, and G. Li, "MV-Sports: a motion and vision sensor integration-based sports analysis system," Computer Communications, pp. 1070-1078, 2018.

[6] H. Araki, H. Fukuda, and T. Motoki, "HADO as techno sports was born by the fusion of IT technology and sports," ReVo, pp. 36-40, 2017.

[7] Z. Wang, J. Li, J. Wang et al., "Inertial sensor-based analysis of equestrian sports between beginner and professional riders under different horse gaits," IEEE Transactions on Instrumentation and Measurement, vol. 67, no. 11, pp. 26922704, 2018.

[8] J. Zhang, Y. Cao, M. Qiao et al., "Human motion monitoring in sports using wearable graphene-coated fiber sensors," Sensors and Actuators A: Physical, vol. 274, pp. 132-140, 2018.

[9] S. Li, B. Zhang, P. Fei, P. M. Shakeel, and R. D. J. Samuel, "Computational efficient wearable sensor network health monitoring system for sports athletics using IoT," Aggression and Violent Behavior, p. 101541, 2020.

[10] Y. Wang, M. Chen, X. Wang, R. H. M. Chan, and W. J. Li, "IoT for next-generation racket sports training," IEEE Internet of Things Journal, vol. 5, no. 6, pp. 4558-4566, 2018.

[11] E. Polak, J. Kulasa, and A. VencesBrito, "Motion analysis systems as optimization training tools in combat sports and martial arts," Revista de Artes Marciales Asiáticas, vol. 10, no. 2, pp. 105-123, 2015.

[12] T. Ray, J. Choi, J. Reeder et al., "Soft, skin-interfaced wearable systems for sports science and analytics," Current Opinion in Biomedical Engineering, vol. 9, pp. 47-56, 2019.

[13] Y. Wei, L. Jiao, S. Wang, R. Bie, Y. Chen, and D. Liu, "Sports motion recognition using MCMR features based on interclass symbolic distance," International Journal of Distributed Sensor Networks, vol. 12, no. 5, 2016.

[14] K. Xia, H. Wang, M. Xu, Z. Li, S. He, and Y. Tang, "Racquet sports recognition using a hybrid clustering model learned from integrated wearable sensor," Sensors, vol. 20, no. 6, p. 1638, 2020.

[15] M. R. Heath, J. J. Janosky, A. Pegno, J. M. Schachne, and P. D. Fabricant, "Age is more predictive of safe movement patterns than are physical activity or sports specialization: a prospective motion analysis study of young athletes," The American Journal of Sports Medicine, vol. 49, no. 7, pp. 1904-1911, 2021.

[16] B. Pueo, "High speed cameras for motion analysis in sports science," Journal of Human Sport and Exercise, vol. 11, no. 1, pp. 53-73, 2016.

[17] M. V. José Mendes Jr. and S. S. Marcelo Pires Jr., "Sensor fusion and smart sensor in sports and biomedical applications," Sensors, vol. 16, no. 10, p. 1569, 2016.

[18] S. Feng and L. Tan, "Simulation of sports and health big data system based on FPGA and Internet of Things," Microprocessors and Microsystems, p. 103416, 2020.

[19] L. Zhang, L. Yang, Z. Wang, and D. Yan, "Sports wearable device design and health data monitoring based on wireless internet of things," Microprocessors and Microsystems, p. 103423, 2020. 
[20] C. Jiao and M. Tang, "The design of sports energy expenditure measuring device based on three-dimensional motion sensor," Journal of Physics: Conference Series, vol. 1423, no. 1, article 012048, 2019.

[21] Y. Jiang, "Combination of wearable sensors and internet of things and its application in sports rehabilitation," Computer Communications, vol. 150, pp. 167-176, 2020. 